Review

\title{
Implication of the hepatokine, fibrinogen-like protein 1 in liver diseases, metabolic disorders and cancer: The need to harness its full potential
}

\author{
Xi-Hua Liu ${ }^{1}$, Lian-Wen $\mathrm{Qi}^{2}$, Raphael N. Alolga ${ }^{1,2}{ }^{\bowtie}$, Qun Liu ${ }^{1 凶}$ \\ 1. State Key Laboratory of Natural Medicines, School of Traditional Chinese Pharmacy, China Pharmaceutical University, Nanjing 210009, China. \\ 2. Clinical Metabolomics Center, China Pharmaceutical University, Nanjing 211198, China. \\ $\triangle$ Corresponding authors: Dr. Qun Liu, State Key Laboratory of Natural Medicines, China Pharmaceutical University, No. 24 Tongjia Lane, Nanjing 210009 , \\ China, E-mail: liuquncpu@126.com, Tel./Fax: +86 25 83271382; Dr. Raphael N. Alolga, School of Traditional Chinese Pharmacy, China Pharmaceutical \\ University, No. 639 Longmian Road, Nanjing 211198, China, E-mail: anammahime@gmail.com, Tel./Fax: +86 2586185983. \\ (c) The author(s). This is an open access article distributed under the terms of the Creative Commons Attribution License (https://creativecommons.org/licenses/by/4.0/). \\ See http://ivyspring.com/terms for full terms and conditions.
}

Received: 2021.09.06; Accepted: 2021.10.24; Published: 2022.01.01

\begin{abstract}
Fibrinogen-like protein 1 (FGL1) is a novel hepatokine that forms part of the fibrinogen superfamily. It is predominantly expressed in the liver under normal physiological conditions. When the liver is injured by external factors, such as chemical drugs and radiation, FGLI acts as a protective factor to promote the growth of regenerated cells. However, elevated hepatic FGLI under high fat conditions can cause lipid accumulation and inflammation, which in turn trigger the development of non-alcoholic fatty liver disease, diabetes, and obesity. FGL1 is also involved in the regulation of insulin resistance in adipose tissues and skeletal muscles as a means of communication between the liver and other tissues. In addition, the abnormally changed FGLI levels in the plasma of cancer patients make it a potential predictor of cancer incidence in clinical practice. FGL1 was recently identified as a major functional ligand of the immune inhibitory receptor, lymphocyte-activation gene 3 (LAG3), thus making it a promising target for cancer immunotherapy except for the classical programmed cell death protein 1/programmed cell death ligand 1 (PD-1/PD-LI) axis. Despite the potential of FGL1 as a new cancer biomarker and therapeutic target, there are few related studies and much of what has been reported are superficial and lack depth and particularity. Therefore, elucidating the role and underlying mechanisms of FGL1 could be crucial for the development of promising diagnostic and therapeutic strategies for related diseases. Here, we provide a comprehensive review of the cellular mechanisms and clinical prospects of FGL1 in the prevention and treatment of liver diseases, metabolic disorders and cancer, and proffer suggestions for future studies.
\end{abstract}

Key words: FGL1; liver diseases; metabolic disorders; cancer; immunotherapy

\section{Background}

Fibrinogen-like protein 1 (FGL1), also called hepatocyte-derived fibrinogen-related protein 1 (HFREP-1) or hepassocin (HPS), belongs to the fibrinogen family with mitochondrial promoter activity. FGL1 is a $68 \mathrm{kD}$ homodimer protein consisting of two $34 \mathrm{kD}$ disulfide bonds, and about 312 amino acids [1, 2]. The genetic code is located on chromosome 8p22-21.3 in the human genome [3]. FGL1 was initially considered as a significantly changed protein in hepatoma cells and subsequently cloned from the human hepatocellular carcinoma (HCC) specimen cDNA library [4]. Similar to fibrinogen, FGL1 has a hydrophobic leader peptide and a characteristic amino acid of the carboxyl terminus with $\beta$-and $\gamma$-subunits [4]. However, due to the lack of platelet-binding sites, cross-linking sites, and thrombin-sensitive sites that are required for blood clotting, FGL1 does not exhibit the classic coagulation function which is characteristic of other fibrinogen members [4].

FGL1 is mainly synthesized in parenchymal hepatocytes, but not in endothelial cells [1]. With respect to its distribution in various tissues, dot blot analysis of human tissues showed that FGL1 was strongly expressed in adult liver, weakly expressed in the pancreas, and not expressed in other tissues [2]. 
Subsequently, FGL1 was also detected in the brown and white adipose tissues of mice, mediating the cross-talk between an injured liver and adipose tissues [5]. As a secretory protein, FGL1 also exists in the bloodstream [6]. It is reported that approximately $20 \%$ FGL1 in plasma is in a free unbound state [7], an indication that the biological function(s) of FGL1 extend(s) far beyond the hepatocytes. Currently, Oncomine database and Lung Cancer Genome Mapping database have shown that FGL1 mRNA is upregulated in lung cancer, prostate cancer, melanoma, and colorectal cancer tissues, but downregulated in pancreatic cancer, liver cancer and head and neck cancer, thereby indicating the involvement of FGL1 in these pathological states [8].

Several studies have suggested the crucial involvement of FGL1 in liver regeneration, cell proliferation, glucose and lipid metabolism, and immune activation. As a liver protective factor, FGL1 accelerates the growth of liver cells by promoting mitochondrial mitosis in the event of liver injury. As a novel functional ligand of lymphocyte-activation gene 3 (LAG3), FGL1 can promote the proliferation of tumor cells [8]. Effective inhibition of the FGL1/LAG3 axis can activate tumor $\mathrm{T}$ cell immunity, which provides a meaningful direction for tumor immunotherapy. Additionally, FGL1 appears to be an atypical biomarker for nonalcoholic fatty liver disease, type 2 diabetes and obesity, and is involved in regulation of adipogenesis, gluconeogenesis, and insulin resistance through diverse molecular mechanisms [9]. We discuss here, the role of FGL1 in liver diseases, metabolic disorders and cancer. We also highlight how these functions could be harnessed for optimum therapeutic outcome.

\section{Role of FGL1 in liver diseases}

\section{FGL1 in liver injury}

The liver is an organ with remarkable regenerative ability. A healthy liver can initiate and regulate self-regeneration through an interplay between several cytokines and growth factors secreted by itself or other organs after resection or moderate injury [10, 11]. FGL1 was found to be specifically upregulated during liver regeneration after damage by D-galactose, and exerts it effects by promoting DNA synthesis [1]. Human FGL1 (hFGL1) corresponding to rat FGL1 was cloned and demonstrated to stimulate cell uptake of ${ }^{3} \mathrm{H}$-thymine and increased DNA synthesis in various primary animal hepatocytes, indicating the role of hFGL1 in promoting cell proliferation [2]. Following the isolation of mouse fibrinogen-related protein-1 (MFREP-1), a mouse homologue of hFGL1, MFREP-1
mRNA was found to be significantly increased after $70 \%$ liver resection in mice, suggesting the role of FGL1 in regulating cell growth during liver regeneration [12]. As a potential therapeutic target for fulminant hepatic failure, administration of FGL1 to rats can alleviate the degree of injury and mortality rate in D-galactose and $\mathrm{CCl}_{4}$-induced liver injury [13]. Conversely, silencing of FGL1 in vivo can aggravate D-galactose-induced liver injury [13]. Besides chemical-induced damage, hepatic and plasma FGL1 was also elevated in response to radiation-induced liver injury [14]. In brief, FGL1 exerts a net expansionary effect on cell growth during liver regeneration as a liver-specific mitogenic growth factor.

Mechanism-wise, it was discovered that the point of action of FGL1 in promoting liver regeneration is mainly between $784 \mathrm{bp}-614 \mathrm{bp}$ in the luciferase-FGL1 promoter. The transcription factors, signal transducer and activator of transcription (STAT3) and hepatocyte nuclear factor 1 (HNF1) controlled the hepatocyte-specific FGL1 transcription after hepatectomy [15]. Moreover, FGL1-specific receptor exists on the membranes of the human hepatic cell line L02, and FGL1 induces phosphorylation of extracellular signal-regulated kinase 1/2 (ERK1/2) through the autocrine route to promote the proliferation of hepatocytes [16]. Further mechanistic exploration revealed the activation of the epidermal growth factor receptor (EGFR) as an important step in FGL1-induced ERK signaling [17]. In hyperglycemic crisis, high glucose increase STAT3 and protein phosphatase 2A (PP2A)-HNF1 activity, and further induced FGL1 expression [18]. Therefore, FGL1 can ameliorate streptozotocin-induced hyperglycotoxicity in mice by increasing the body's antioxidant capacity [18].

In conclusion, FGL1 contributes to the self-repair process of an injured liver. When the liver is exposed to chemical drugs, radiation or hyperglycemic crisis, FGL1 levels are significantly elevated, a process that may depend on the binding of transcription factor STAT3 and HNF1 in the FGL1 gene promoter. Furthermore, FGL1 binds to the membrane-specific receptor of hepatocytes and induces cell proliferation through an autocrine mechanism, which is dependent on the EGFR/ERK/tyrosine protein kinase (Src) pathway. In addition, liver injury also enhances the expression of FGL1 in brown adipose tissues suggesting a cross-talk between the injured liver and adipose tissues [5].

\section{FGL1 in nonalcoholic fatty liver disease}

Nonalcoholic fatty liver disease (NAFLD) is a leading cause of liver-related morbidity and mortality 
[19]. Hepatocytes secrete $\sim 560$ hepatokines, many of which affect metabolism both locally and in distant organs and are linked to the pathogenesis of metabolic diseases [20,21]. A total of 393 subjects with $(n=194)$ or without $(n=199)$ NAFLD were enrolled in a study cohort to evaluate the serum FGL1 concentration. After an overnight 12 -h fast, all subjects received biochemical blood tests. NAFLD subjects had a significantly higher serum FGL1 level than those without NAFLD $(7699.7 \pm 194.9$ vs. $6112.3 \pm$ $143.7 \mathrm{lg} / \mathrm{ml} ; p$ <0.001) [22]. In addition, serum FGL1 concentrations were elevated in diabetic patients with NAFLD $(n=50)$ compared with the diabetic group (n = 50) [23]. FGL1 was significantly increased in high fat diet (HFD)-fed mice and primary hepatocytes treated with oleic acid, both of which constituted the in vivo and in vitro models of NAFLD [22]. FGL1 silencing or overexpression consequently alleviated or worsened NAFLD as evinced by indices such as serum transaminase, triglyceride levels, inflammatory markers and lipid synthesis-related genes (sterol regulatory element-binding protein-1, fatty acid synthase and acetyl-CoA carboxylase-1) [22]. Mechanistically, there are potential binding sites of STAT3 in the FGL1 gene promoter, and unsaturated fatty acid activates STAT3 to increase FGL1 expression. The blockade of STAT3 dose- dependently inhibited unsaturated fatty acid induced FGL1 expression, which accounts for the increased level of FGL1 under proinflammatory conditions [24]. In addition, FGL1 regulates NAFLD through an ERK1/2-dependent pathway [22]. FGL1 affects the phosphorylation of ERK1/2 to increase the activity of lipid synthesis-related genes and further induces lipogenesis. In contrast, PD98059, the ERK1/2 inhibitor, blocked FGL1-induced synthesis-related genes expression in vitro [22].

On the basis of the aforementioned, FGL1 may be an acute reactant of liver steatosis stress, and participates in hepatic lipid metabolism in an ERK1/2-dependent manner. FGL1 can therefore serve as a biomarker candidate in the pathogenesis of NAFLD and as an indicator of treatment response.

\section{Role of FGL1 in metabolic diseases}

\section{FGLI in type 2 diabetes}

It was reported that plasma FGL1 levels in diabetic patients were significantly higher than the normal population, but multiple linear regression analysis showed that the elevated FGL1 was independently associated with fasting glucose levels, insulin resistance, impaired fasting glucose, impaired glucose tolerance and newly diagnosed diabetes [25, 26]. FGL1 null mice exhibited fasting hyperglycemia and enhanced hepatic glucose production [25]. FGL1 plays a crucial role in the development of insulin resistance by regulating ERK1/2 activity, indicating that it may be a risk factor for prediabetes, and could be a potential diagnostic marker of diabetes [25].

The occurrence of diabetes is closely related to NAFLD. A recent meta-analysis has shown that NAFLD increases the risk of type 2 diabetes by about 2-fold [27]. The hepatokines could be therapeutic targets for both NAFLD and diabetes [28]. The relationship between FGL1 and liver steatosis in diabetic patients with or without NAFLD was investigated and a significant increase of serum FGL1 in type 2 diabetics and NAFLD patients, providing evidence that FGL1 elevation in both diseased conditions may facilitate hepatic lipid accumulation [28].

\section{FGL1 in obesity}

Obesity is the consequence of metabolic disorders in multiple organs [29]. The liver plays a central role in regulating energy balance by sensing nutrient availability and altering metabolite or energy production from various organ systems, including the central nervous system, adipose tissues, and skeletal muscles. The secretory hepatokines are critical in influencing metabolic phenotypes through inter-organ communication [30]. Recently, the relationship between FGL1 and obesity was confirmed in obese individuals. The plasma FGL1 of obese subjects was higher than that of healthy individuals, and univariate analysis confirmed that BMI, waist circumference, total fat, visceral fat, and subcutaneous fat area were all positively correlated with FGL1 level [31]. FGL1 null mice were shown to have abnormal plasma lipid profiles, fasting hyperglycemia and exhibited differences in white and brown adipose tissue morphology [5]. Mechanismwise, Jeong et al. found that palmitate induced FGL1 expression in primary hepatocytes through endoplasmic reticulum (ER) stress and p38-mediated pathway, and also triggered the transcriptional activation of FGL1 via binding of CCAAT/enhancerbinding protein $\beta(C / E B P \beta)$ to its promoter [32]. Skeletal muscle is the main site of glucose utilization. Jeong et al. found that FGL1 mainly responded to hyperlipidemic environment through the phosphorylation of c-Jun N-terminal kinase (JNK), and further impaired insulin sensitivity through EGFR-mediated pathway, but did not affect inflammatory response and ER stress in skeletal muscles [32].

FGL1 is a communication factor that transmits signals of liver metabolic disorders to other tissues. Changes in FGL1 levels in the liver not only lead to 
hepatic metabolic disorders, but also affect obesityrelated insulin resistance in adipose tissues and skeletal muscles. As a potential diagnostic biomarker and indicator of NAFLD, diabetes and obesity treatment response, FGL1 is a candidate target worthy of further study at the mechanistic level in the continual search for therapeutic remedies for these diseases.

\section{Role of FGL1 in cancer}

\section{FGLI in liver cancer}

FGL1 is a highly expressed protective hepatocyte mitogen in the liver after resection. Studies have shown that FGL1 is underexpressed in hepatocellular carcinoma (HCC) at both the protein and mRNA levels [3]. Mice that lack FGL1 develop HCC at a rate more than twice that of wild type mice treated with diethyl nitrosamine. Consistent with these findings, hepatocellular cancers from FGL1 knockout mice grew faster than those from the wild type mice [33]. Furthermore, the level of FGL1 in HCC was found to be linked to the degree of cell differentiation, as poorly differentiated HCCs tend to have lower levels of FGL1 compared to highly differentiated HCCs [3].

The possible reasons for the persistent low levels of FGL1 in the development of liver cancer have also been provided by some groups. Heterozygosity loss analysis of liver cancer specimen showed that $57.1 \%$ of HCCs have allele loss of the FGL1 gene on chromosome 8p22 [3], a position associated with HCC progression and recurrence for frequently lost isopet genes [34]. HNF1a acts as an important liver-specific cis-acting element for FGL1, and downregulation of HNF1a might responsible for the inhibition of FGL1 transcription in liver cancer [15]. Mechanismwise, endogenous HNF1a binds to high mobility group box-1 protein (HMGB1) and cAMP-response element binding protein (CREB), and activates FGL1 promoter by interacting with the $470-457 \mathrm{bp}$ fragment of the FGL1 upstream promoter [15]. FGL1 also acts as a tumor suppressor in HCC through protein kinase $\mathrm{B} /$ mammalian target of rapamycin (Akt/mTOR) signaling [33], a critical pathway in the development and progression of HCC [35].

Interestingly, the paradoxical role of FGL1 in normal and cancer cells proliferation were partly explained by Cao et al. They showed that there is a signal-peptide dependent pattern for FGLI in liver-derived cells. It acts as a key positive regulator in non-tumor cells via autocrine signaling and a tumor suppressor in HCC cells through intracrine signaling [16].

\section{FGL1 in gastric, lung and other cancers}

FGL1 was reported to be up-regulated in gastric cancer (GC) tissues and the survival time of GC patients with high FGL1 levels was markedly shorter than the patients with low FGL1 levels [36]. Hence, high expression of FGL1 can be an independent predictor of poor prognosis for GC patients [36].

In addition, FGL1 is involved in the epithelial-mesenchymal transformation (EMT), which is activated in the malignant progression of cancer and plays an important role in cancer development [37]. The most common type of lung cancer is non-small cell lung cancer (NSCLC), which accounts for about $85 \%$ of lung cancer cases [38]. Liver kinase B1 (LKB1) controls the initiation, differentiation and metastasis of lung cancer cells, and is a key barrier to the development and progression of lung cancer [39]. FGL1 was found to be significantly increased in LKB1 mutated lung adenocarcinoma [40]. Silencing FGL1 was found to be a novel approach for the treatment of LKB1 mutated lung adenocarcinoma by inducing EMT and angiogenesis [40]. EGFR, which is expressed in more than $60 \%$ of NSCLC, has become an important target for the treatment of NSCLC [41]. FGL1 was also significantly upregulated in EGFR mutant NSCLC cells. Gefitinib is the first-line treatment for patients with advanced NSCLC with activating EGFR mutation. FGL1 was found to be highly expressed in gefitinib-resistant NSCLC cell line [42]. The decreased signal for poly(ADP-ribose) polymerase 1 (PARP1)-caspase 3 pathway induced by silencing FGL1 may be the underlying mechanism for gefitinib-resistant cell apoptosis [42].

Wang et al. conducted a meta-analysis of the Oncomine databases to map the level of FGL1 in a series of solid tumors, and found FGL1 is highly expressed in lung cancer, prostate cancer, breast cancer, melanoma and colorectal cancer, but downregulated in pancreas cancer, head and neck cancers [8].

\section{FGLI in cancer immunotherapy}

Immunotherapy is a type of cancer treatment that helps the immune system to fight cancer. Immune checkpoint blockade therapy marked the beginning of a new era of immunotherapy. It uses monoclonal antibodies to directly target the antigens, and block the pathways involved in T cell inhibition, such as cytotoxic $\mathrm{T}$ lymphocyte antigen-4 (CTLA4) and programmed cell death protein 1 (PD-1) [43]. LAG3 is a promising next-generation immune checkpoint and its blockade is actively pursued in clinical trials [44]. As a receptor, LAG3 can transmit immunosuppressive signals and negatively regulate the proliferation and activation of CD4+ and CD8+ T cells [44]. The emergence of FGL1 as a major functional ligand of LAG3 has attracted great 
attention in the field of tumor immunotherapy. FGL1 was found strongly binds to LAG3 on the surface of T cells, and the interaction of these two proteins is independent of MHC class II, a canonical ligand of LAG3 [8]. Tumor growth in LAG3 or FGL1 knockout mice was suppressed after colon cancer cells transplantation and by further blocking the interaction between FGL1 and LAG3, stimulated the activation and proliferation of $\mathrm{T}$ cells in the tumor microenvironment, thus improving immunity and the elimination of tumors [8].

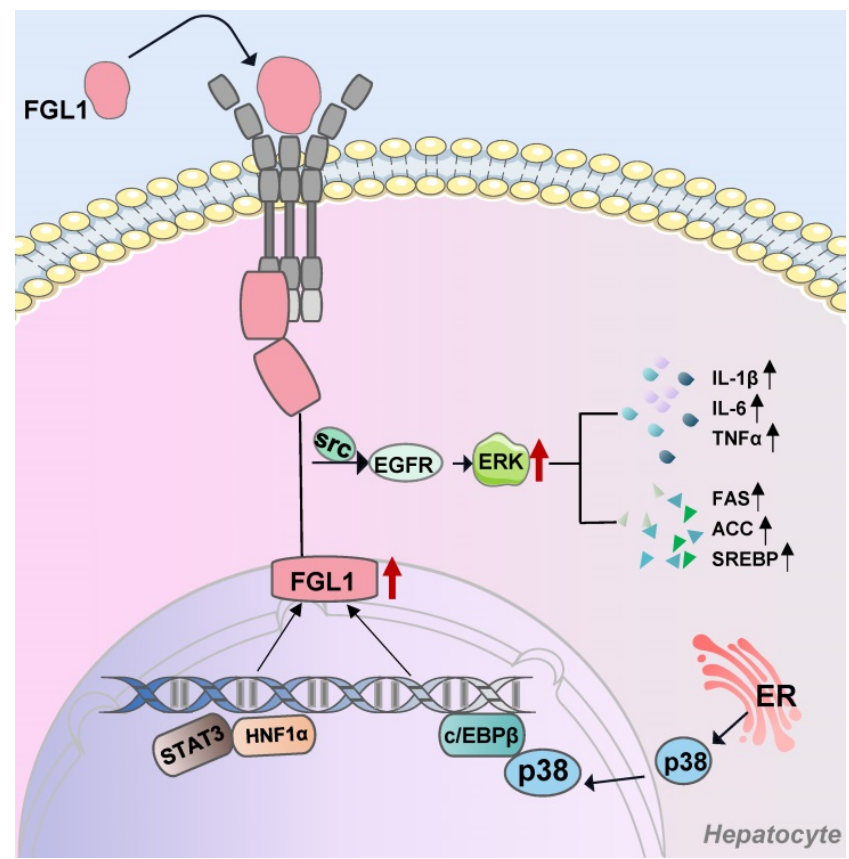

Figure 1. FGL1-involved signaling pathways in hepatocytes. Extracellular FGL1 binds to specific hepatocytes membrane receptors and enters the cells. Transcription factors STAT3, HNF1 $\alpha$, and C/EBP $\beta$ bind to the intracellular FGL1 promoter region to promote its transcriptional activation. Elevated FGL1 exerts its biological functions through the EGFR/SRC/ERK cascade. FGL1: fibrinogen-like protein 1; Src: tyrosine protein kinase; EGFR: epidermal growth factor receptor; ERK: extracellular signal-regulated kinase; p38: mitogen-activated protein kinase; STAT3: signal transducer and activator of transcription 3; HNF1 $\alpha$ : hepatocyte nuclear factor 1 alpha; c/EBP $\beta$ : CCAAT/enhancer-binding protein $\beta$; ER: endoplasmic reticulum.

Blockade of the FGL1/LAG3 pathway has been exploited by many a researcher as an immunotherapeutic route for cancers. FGL1/LAG3 blockade combined with natural products, seems to be more effective against liver cancer. Oxysophocarpine is a known alkaloid with demonstrable anti-HCC properties both in vivo and in vitro by reducing FGL1 expression and sensitizing CD8 $+\mathrm{T}$ cells to LAG3 immunotherapy [45]. Blockade of the FGL1/LAG3 axis with concurrent administration of metformin can enhance T-cell-mediated immune response, improve the tumor microenvironment immunosuppression, and enhance general anti-tumor immunity [46].

NSCLC patients with high FGL1 expression have poor outcomes when treated with PD-1/programmed cell death ligand 1 (PD-L1) inhibitors. Intriguingly, when FGL1/LAG3 and PD-1/PD-L1 axes were simultaneously inhibited, the therapeutic effect was significantly improved, evidenced by a longer lifespan and reduced tumor burden in mice [42]. In addition, the combination of PD-1/PD-L1 signaling blockade and FGL1 gene silencing showed a high synergistic effect in the treatment of breast cancer [46]. Together, the FGL1/LAG3 and PD-1/PD-L1 axes might be independently targeted in treating cancers though synergism of the two yields better therapeutic outcomes.

\section{Role of FGL1 in rheumatoid arthritis}

Rheumatoid arthritis (RA) is a common, chronic, systemic autoimmune inflammatory disease. Early diagnosis of RA is crucial in combating the long term irreversible pathophysiological damage that would otherwise occur [47]. In a proteomic study involving 1244 clinical samples, FGL1 levels were 10-time higher in RA patients than in healthy subjects, and acted as one of the most differentially expressed immune/inflammation-related proteins in RA patients with moderate and high disease activity [48]. Thus, FGL1 has emerged as a novel protein capable of predicting disease progression in RA patients before and after treatment.

\section{Conclusions and future directions}

\section{Harnessing the potential of FGLI as a hepatokine}

The liver, a key organ that regulates energy homeostasis in the human body, secretes proteins called hepatokines, which play crucial roles in diverse medical conditions such as insulin resistance [49]. The biological roles of hepatokines in systemic communication between the liver and other organs is a rapidly developing field and could become a scientific hotspot [50,51]. As a hepatokine, extracellular FGL1 binds to specific hepatocytes membrane receptors and enters the cells. Otherwise, transcription factors STAT3, HNF1 $\alpha$, and C/EBP $\beta$ bind to the intracellular FGL1 promoter region to promote its transcriptional activation. In addition, elevated FGL1 exerts its biological functions through the EGFR/Src/ERK cascade (Figure 1). FGL1 also acts as a transmitter of communication between the liver, skeletal muscles, and adipose tissues, as early on outlined and schematically summarized in Figure 2. In brief, elevated FGL1 in the liver under external stimulation promotes DNA synthesis, inhibits ROS production, and causes insulin resistance, steatosis and inflammation. Excess FGL1 also causes obesity-related insulin resistance in skeletal muscle 
through the EGFR/JNK mediated pathway. In addition, high levels of FGL1 induce adipogenesis through an ERK1/2-C/EBP $\beta$-dependent pathway in adipocytes.

Taking due cognisance of the aforementioned cardinal pathways and the interplay that exists between them, the medical significance of the hepatokine, FGL1 cannot be overemphasized. There is therefore need to further explore the known as well as search for newer mechanistic pathways that can be harnessed for therapeutic benefits.

\section{Harnessing the prospects of FGLI as a therapeutic target}

Among the immunodetection sites discovered so far, the inhibitory effect of PD-1/PD-L1 on tumor development has been most thoroughly studied. Clinically, the effective rate of PD-1/PD-L1 inhibitors is approximately $80 \%$ in lymphoma, $60 \%$ in high microsatellite instability tumors, and $10 \% \sim 30 \%$ in other common solid tumors [52], thereby limiting its wide-scale applicability. Therefore, it is very necessary to search for immune escape mechanisms other than the PD-1/PD-L1 route. FGL1 has recently been identified as a ligand of LAG3, providing scientific evidence for same as a possible therapeutic target. The discovery of immunotherapy via the LAG3/FGL1 axis represents a milestone in cancer treatment. Patients who do not respond to
PD-1/PD-L1 inhibitors may have access to alternative therapies. It would be worthwhile investigating the simultaneous blockade of both PD-1/PD-L1 and FGL1/LAG3 axes in further research.

As earlier indicated, FGL1 might be a vital marker in cancer initiation and progress. A summary of all reported FGL1-mediated effects in cancers is summarized in Table 1. More emphasis could be laid on monoclonal antibodies or small interfering RNA associated with FGL1, and/or as combination therapy with first-line drugs or natural products with proven anticancer properties as alternatives. These traditional remedies either used holistically or on a singlecomponent basis, could be investigated in the context of further exploring FGL1 as the therapeutic target in different cancers.

In conclusion, we have provided an overview of the roles of FGL1 in various diseases in this review (pictorially summarized in Figure 3). On the basis of available literature, FGL1 is vest with two key functional identities, as a hepatokine and a therapeutic target. The former plays a hepatoprotective role in liver regeneration and self-repair after injury, as well as a communicator between different organs in metabolic diseases. The latter identity presents FGL1 as a possible novel diagnostic biomarker or therapeutic target for cancers and various metabolic-associated diseases.

Table 1. Functional roles of FGL1 in different types of cancer

\begin{tabular}{|c|c|c|c|c|}
\hline Cancer types & $\begin{array}{l}\text { FGL1 } \\
\text { levels }\end{array}$ & Study subjects & Key messages & Ref. \\
\hline \multirow[t]{4}{*}{ Liver cancer } & \multirow[t]{4}{*}{$\downarrow$} & $\begin{array}{l}\text { Human solid tumors } \\
\text { and human HCC cell lines }\end{array}$ & $\begin{array}{l}\text { The level of FGL1 in HCC cells is closely related to tumor progression and degree of differentiation, } \\
\text { and the suppression of FGL1 enhances HCC cell growth. }\end{array}$ & {$[3]$} \\
\hline & & Mouse xenotransplant & $\begin{array}{l}\text { Knockout of FGL1 resulted in HCC cell proliferation, reducing the incidence of HCC in an } \\
\text { Akt/mTOR dependent manner. }\end{array}$ & [33] \\
\hline & & HepG2 cells and mouse & $\begin{array}{l}\text { Down-regulation of HNF1a causes, at least in part, the transcriptional down-regulation of FGL1 in } \\
\text { HCC. }\end{array}$ & [15] \\
\hline & & Human solid tumors & $\begin{array}{l}\text { Meta-analysis of the Oncomine databases revealed the downregulation of FGL1 mRNA in human } \\
\text { liver cancer solid tumors. }\end{array}$ & {$[8]$} \\
\hline \multirow[t]{3}{*}{ Lung cancer } & \multirow[t]{3}{*}{$\uparrow$} & Human solid tumors & $\begin{array}{l}\text { Loss of FGL1 may be a novel approach to promote EMT and angiogenesis in patients with LKB1 } \\
\text { mutant lung adenocarcinoma. }\end{array}$ & [8] \\
\hline & & $\begin{array}{l}\text { PC9 or PC9/GR cells and } \\
\text { mouse xenotransplant }\end{array}$ & $\begin{array}{l}\text { Loss of FGL1 can improve the acquired resistance of gefitinib in NSCLC via PARP1/caspase } 3 \\
\text { pathway. }\end{array}$ & {$[42]$} \\
\hline & & Human soild tumors & $\begin{array}{l}\text { Meta-analysis of the Oncomine databases revealed the upregulation of FGL1 mRNA in human lung } \\
\text { cancer solid tumors, with the highest percentage of upregulation (35\%) in lung cancer datasets. }\end{array}$ & [8] \\
\hline $\begin{array}{l}\text { Gastric } \\
\text { cancer }\end{array}$ & $\uparrow$ & Human solid tumors & FGL1 has the potential to be a predictor in GC patients as well as a target for the treatment of GC. & [36] \\
\hline Breast cancer & $\uparrow$ & Mouse xenotransplant & $\begin{array}{l}\text { Targeted delivery of siFGL1 and metformin can inhibit the growth and migration of breast cancer } \\
\text { cells. }\end{array}$ & [8] \\
\hline $\begin{array}{l}\text { Prostate } \\
\text { cancer }\end{array}$ & $\uparrow$ & Human solid tumors & $\begin{array}{l}\text { Meta-analysis of the Oncomine databases and TCGA cancer database revealed the upregulation of } \\
\text { FGL1 mRNA in human prostate cancer solid tumors. }\end{array}$ & [8] \\
\hline $\begin{array}{l}\text { Melanoma } \\
\text { cancer }\end{array}$ & $\uparrow$ & Human solid tumors & $\begin{array}{l}\text { Meta-analysis of the Oncomine databases revealed the upregulation of FGL1 mRNA in human } \\
\text { melanoma solid tumors. }\end{array}$ & [8] \\
\hline $\begin{array}{l}\text { Colorectal } \\
\text { cancer }\end{array}$ & $\uparrow$ & Human solid tumors & $\begin{array}{l}\text { Meta-analysis of the Oncomine databases revealed the upregulation of FGL1 mRNA in human } \\
\text { colorectal cancer solid tumors. }\end{array}$ & [8] \\
\hline $\begin{array}{l}\text { Pancreas } \\
\text { cancer }\end{array}$ & $\downarrow$ & Human solid tumors & $\begin{array}{l}\text { Meta-analysis of the Oncomine databases revealed the downregulation of FGL1 mRNA in human } \\
\text { pancreas solid tumors, with } 60 \% \text { downregulation. }\end{array}$ & {$[8]$} \\
\hline $\begin{array}{l}\text { Head and } \\
\text { neck cancers }\end{array}$ & $\downarrow$ & Human solid tumors & $\begin{array}{l}\text { Meta-analysis of the Oncomine databases revealed the downregulation of FGL1 mRNA in human } \\
\text { head and neck cancers solid tumors. }\end{array}$ & [8] \\
\hline
\end{tabular}




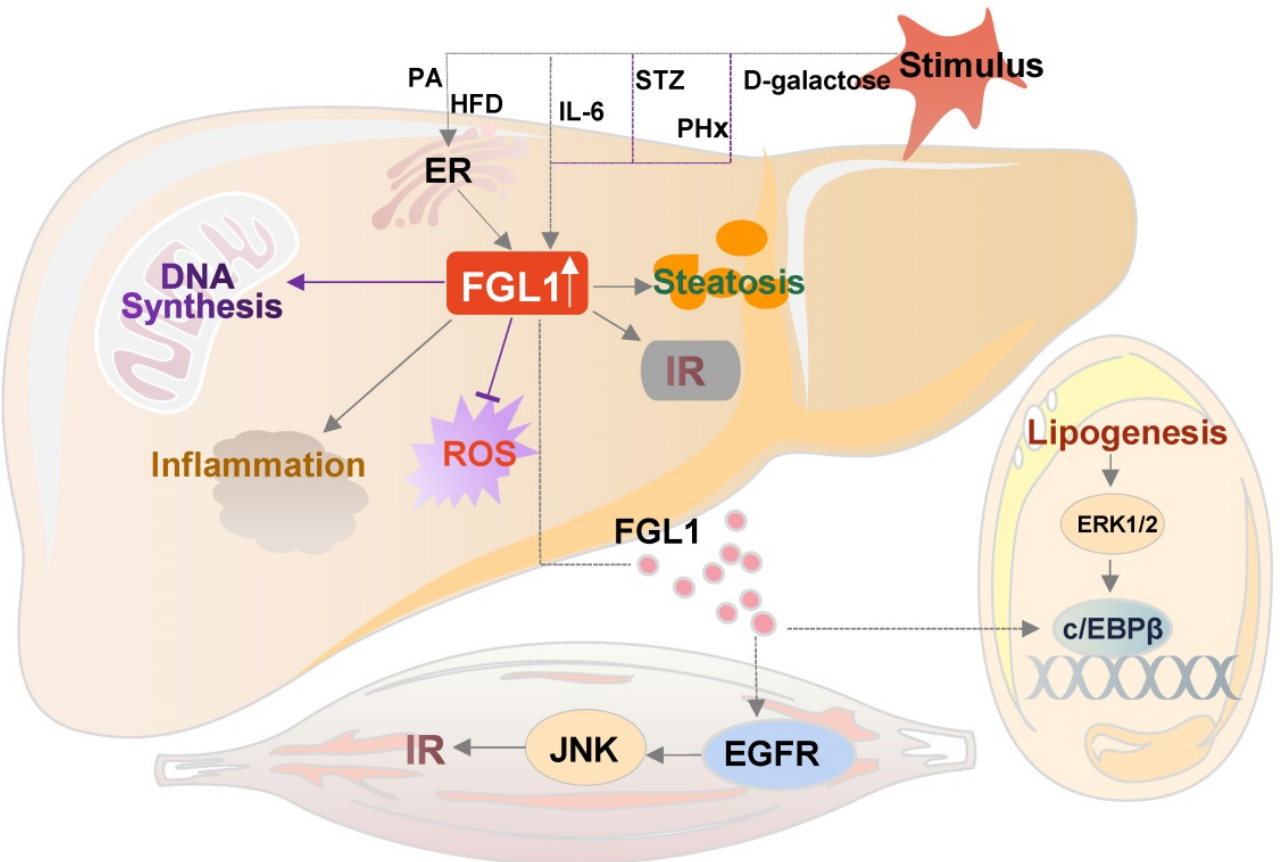

Figure 2. The crosstalk of FGLI in metabolic diseases. Elevated FGLI in the liver promotes DNA synthesis, inhibits ROS production, and causes insulin resistance, steatosis and inflammation. Excess FGL1 causes obesity-related insulin resistance in skeletal muscle through the EGFR/JNK-mediated pathway, and adipogenesis through an ERK1/2-c/EBP $\beta$-dependent pathway in the adipose tissue. HFD: high-fat diet; PA: palmitic acid; IL-6: interleukin 6; STZ: streptozotocin; PHx: partial hepatectomy; ER: endoplasmic reticulum; FGL1: fibrinogen-like protein 1; c/EBP $\beta$ : CCAAT/enhancer-binding protein $\beta$; EGFR: epidermal growth factor receptor; ERK1/2: extracellular signal-regulated kinase 1/2; IR: insulin resistance; JNK: c-Jun N-terminal kinase.

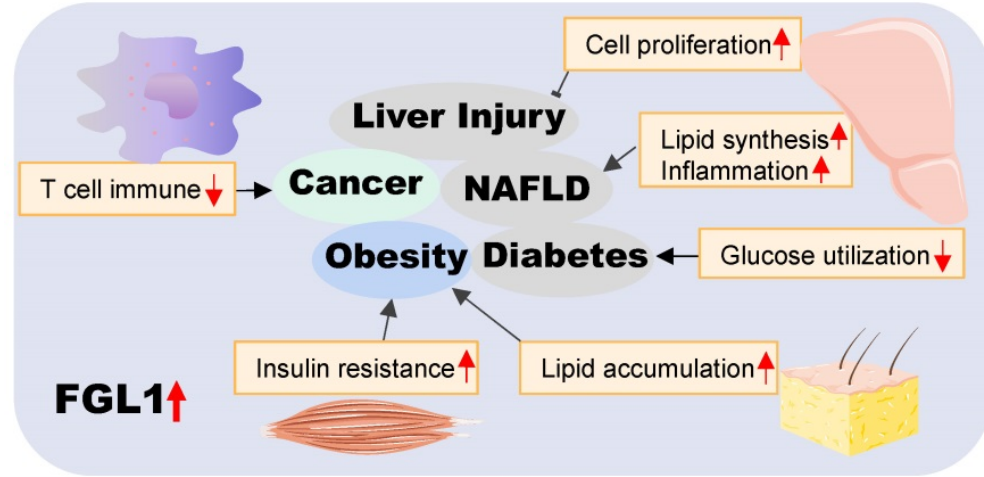

Figure 3. Summary of the role of FGL1 in various diseases. Elevated FGL1 regulates glucose and lipid metabolism, insulin resistance, cell proliferation, and immune response, leading to obesity, diabetes, non-alcoholic fatty liver disease, cancer and other diseases.

\section{Abbreviations}

Akt: protein kinase $\mathrm{B} ; \mathrm{c} / \mathrm{EBP} \beta$ : CCAAT/ enhancer-binding protein $\beta$; CREB: cAMP-response element binding protein; CTLA4: cytotoxic $\mathrm{T}$ lymphocyte antigen-4; ER: endoplasmic reticulum; EMT: epithelial-mesenchymal transformation; ERK1/2: extracellular signal-regulated kinase1/2; EGFR: epidermal growth factor receptor; FGL1: fibrinogen-like protein 1; GC: gastric cancer; HMGB1: high mobility group box-1 protein; HFREP-1: hepatocyte-derived fibrinogen-related protein 1; HNF1 $\alpha$ : hepatocyte nuclear factor 1 alpha; HFD: high fat diet; HPS: hepassocin; HCC: human hepatocellular carcinoma; hFGL1: human FGL1; JNK: c-Jun N-terminal kinase; LAG3: lymphocyte-activation gene
3; LKB1: liver kinase B1; mTOR: mammalian target of rapamycin; MFREP-1: mouse fibrinogen-related protein-1; NSCLC: non-small cell lung cancer; NAFLD: nonalcoholic fatty liver disease; PARP1: poly (ADP-ribose) polymerase 1; PP2A: protein phosphatase 2A; PD-1: programmed death-1; PD-L1: programmed cell death ligand 1; RA: rheumatoid arthritis; STAT3: signal transducer and activator of transcription 3; Src: tyrosine protein kinase.

\section{Acknowledgements}

This work was supported by the Outstanding Youth Scholars Foundation of Jiangsu Province (BK20200082) and National Natural Science Foundation of China (Nos. 81973550 and 82174036). 


\section{Author Contributions}

Xi-Hua Liu, Raphael N. Alolga and Qun Liu prepared the manuscript. Lian-Wen Qi was responsible for overall supervision. All authors reviewed the article critically for intellectual content and agreed to the published version of the manuscript.

\section{Competing Interests}

The authors have declared that no competing interest exists.

\section{References}

1. Hara H, Uchida S, Yoshimura $\mathrm{H}$, et al. Isolation and characterization of a novel liver-specific gene, hepassocin, upregulated during liver regeneration. Biochim Biophys Acta. 2000; 1492: 31-44.

2. Hara H, Yoshimura S, Uchida $Y$, et al. Molecular cloning and functional expression analysis of a cDNA for human hepassocin, a liver-specific protein with hepatocyte mitogenic activity. Biochim Biophys Acta. 2001; 1520: 45-53.

3. Yan J, Yu Y, Wang N, et al. LFIRE-1/HFREP-1, a liver-specific gene, is frequently downregulated and has growth suppressor activity in hepatocellular carcinoma. Oncogene. 2004; 23: 1939-49.

4. Yamamoto $\mathrm{T}$, Gotoh M, Sasaki $\mathrm{H}$, et al. Molecular cloning and initial characterization of a novel fibrinogen-related gene, HFREP-1. Biochem Biophys Res Commun. 1993; 193: 681-7.

5. Demchev V, Malana G, Vangala D, et al. Targeted deletion of fibrinogen like protein 1 reveals a novel role in energy substrate utilization. PLoS One. 2013; 8: e58084.

6. Rijken DC, Dirkx SP, Luider TM, et al. Hepatocyte-derived fibrinogen-related protein-1 is associated with the fibrin matrix of a plasma clot. Biochem Biophys Res Commun. 2006; 350: 191-4.

7. Liu Z, Ukomadu C. Fibrinogen-like protein 1, a hepatocyte derived protein is an acute phase reactant. Biochem Biophys Res Commun. 2008; 365: 729-34

8. Wang J, Sanmamed MF, Datar I, et al. Fibrinogen-like Protein 1 is a Major Immune Inhibitory Ligand of LAG-3. Cell. 2019; 176: 334-47.

9. Kucukoglu O, Sowa JP, Mazzolini GD, et al. Hepatokines and adipokines in NASH-related hepatocellular carcinoma. J Hepatol. 2021; 74: 442-57.

10. Michalopoulos GK, Bhushan B. Liver regeneration: biological and pathological mechanisms and implications. Nat Rev Gastroenterol Hepatol. 2021; 18: 40-55.

11. Clemens MM, McGill MR, Apte U. Mechanisms and biomarkers of liver regeneration after drug-induced liver injury. Adv Pharmacol. 2019; 85: 241-62

12. Yan J, Ying H, Gu F, et al. Cloning and characterization of a mouse liverspecific gene mfrep-1, up-regulated in liver regeneration. Cell Res. 2002; 12: 353-61.

13. Li CY, Cao CZ, Xu WX, et al. Recombinant human hepassocin stimulates proliferation of hepatocytes in vivo and improves survival in rats with fulminant hepatic failure. Gut. 2010; 59: 817-26.

14. Han NK, Jung MG, Jeong YJ, et al. Plasma Fibrinogen-Like 1 as a Potential Biomarker for Radiation-Induced Liver Injury. Cells. 2019; 8: 1042.

15. Yu HT, Yu M, Li CY, et al. Specific expression and regulation of hepassocin in the liver and down-regulation of the correlation of HNF1alpha with decreased levels of hepassocin in human hepatocellular carcinoma. J Biol Chem. 2009; 284: 13335-47.

16. Cao MM, Xu WX, Li CY, et al. Hepassocin regulates cell proliferation of the human hepatic cells L02 and hepatocarcinoma cells through different mechanisms. J Cell Biochem. 2011; 112: 2882-90.

17. Gao M, Zhan YQ, Yu M, et al. Hepassocin activates the EGFR/ERK cascade and induces proliferation of L02 cells through the Src-dependent pathway. Cell Signal. 2014; 26: 2161-6.

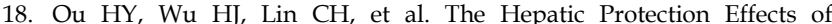
Hepassocin in Hyperglycemic Crisis. J Clin Endocrinol Metab. 2017; 102: 2407-15.

19. Whalley S, Puvanachandra P, Desai A, et al. Hepatology outpatient service provision in secondary care: a study of liver disease incidence and resource costs. Clin Med (Lond). 2007; 7: 119-24.

20. Mouchiroud M, Camiré É, Aldow M, et al. The hepatokine Tsukushi is released in response to NAFLD and impacts cholesterol homeostasis. JCI Insight. 2019; 4: e129492.
21. Lebensztejn DM, Flisiak-Jackiewicz M, Białokoz-Kalinowska I, et al. Hepatokines and non-alcoholic fatty liver disease. Acta Biochim Pol. 2016; 63: 459-67.

22. Wu HT, Lu FH, Ou HY, et al. The role of hepassocin in the development of non-alcoholic fatty liver disease. J Hepatol. 2013; 59: 1065-72.

23. Lu FH, Ou HY, Wu HT, et al. Serum hepassocin concentrations in diabetic patients with or without nonalcoholic fatty liver disease. Diabetes Management. 2014; 4: 255-61.

24. Cheng KP, Ou HY, Hung HC, et al. Unsaturated Fatty Acids Increase the Expression of Hepassocin through a Signal Transducer and Activator of Transcription 3-Dependent Pathway in HepG2 Cells. Lipids. 2018; 53: 863-9.

25. Wu HY, Ou HY, Hung HC, et al. A novel hepatokine, HFREP1, plays a crucial role in the development of insulin resistance and type 2 diabetes. Diabetologia. 2016; 59: 1732-42.

26. Kang Z, Li HY, Ou HY, et al. Role of placental fibrinogen-like protein 1 in gestational diabetes. Transl Res. 2020; 218: 73-80.

27. Jarvis $H$, Craig D, Barker $R$, et al. Metabolic risk factors and incident advanced liver disease in non-alcoholic fatty liver disease (NAFLD): A systematic review and meta-analysis of population-based observational studies. PLoS Med. 2020; 17: e1003100.

28. Abdelmoemen G, Khodeir SA, Zaki AN, et al. Overexpression of Hepassocin in Diabetic Patients with Nonalcoholic Fatty Liver Disease May Facilitate Increased Hepatic Lipid Accumulation. Endocr Metab Immune Disord Drug Targets. 2019; 19: 185-8.

29. Huang S, Liu S, Guo J, et al. The PI3K/AKT pathway in obesity and type 2 diabetes. Int J Biol Sci. 2018; 14: 1483-96.

30. Meex PCR, Watt MJ. Hepatokines: linking nonalcoholic fatty liver disease and insulin resistance. Nat Rev Endocrinol. 2017; 13: 509-20.

31. Huang RL, Li RL, Du YF, et al. Discovery of a role of the novel hepatokine, hepassocin, in obesity. Biofactors. 2020; 46: 100-5.

32. Jung TW, Chung $\mathrm{YH}, \mathrm{Kim} \mathrm{HC}$, et al. Hyperlipidemia-induced hepassocin in the liver contributes to insulin resistance in skeletal muscle. Mol Cell Endocrinol. 2018; 470: 26-33.

33. Nayeb-Hashemi H, Desai A, Demchev V, et al. Targeted disruption of fibrinogen like protein-1 accelerates hepatocellular carcinoma development. Biochem Biophys Res Commun. 2015; 465: 167-73.

34. Di Benedetto $M$, Pineau $P$, Nouet $S$, et al. Mutation analysis of the $8 \mathrm{p} 22$ candidate tumor suppressor gene ATIP/MTUS1 in hepatocellular carcinoma. Mol Cell Endocrinol. 2006; 252: 207-15.

35. Ma L, Ji L, Yu Y, et al. Novel molecular targets for diagnosis and treatment of hepatocellular carcinoma. Discov Med. 2015; 19: 7-14.

36. Zhang Y, Qiao HX, Zhou YT, et al. Fibrinogen-like-protein 1 promotes the invasion and metastasis of gastric cancer and is associated with poor prognosis. Mol Med Rep. 2018; 18: 1465-72.

37. Zhang Y, Weinberg RA. Epithelial-to-mesenchymal transition in cancer: complexity and opportunities. Front Med. 2018; 12: 361-73.

38. Molina JR, Yang P, Cassivi SD, et al. Non-small cell lung cancer: epidemiology, risk factors, treatment, and survivorship. Mayo Clin Proc. 20108; 83: 584-94.

39. Ji H, Ramsey MR, Hayes DN, et al. LKB1 modulates lung cancer differentiation and metastasis. Nature. 2007; 448: 807-10.

40. Bie F, Wang G, Qu X, et al. Loss of FGL1 induces epithelial-mesenchymal transition and angiogenesis in LKB1 mutant lung adenocarcinoma. Int J Oncol. 2019; 55: 697-707.

41. da Cunha Santos G, Shepherd FA, Tsao MS. EGFR mutations and lung cancer. Annu Rev Pathol. 2011; 6: 49-69.

42. Sun C, Gao W, Liu J, et al. FGL1 regulates acquired resistance to Gefitinib by inhibiting apoptosis in non-small cell lung cancer. Respir Res. 2020; 21: 210 .

43. Couzin-Frankel J. Breakthrough of the year 2013. Cancer immunotherapy. Science. 2013; 342: 1432-3.

44. Ruffo E, Wu RC, Bruno TC, et al. Lymphocyte-activation gene 3 (LAG3): The next immune checkpoint receptor. Semin Immunol. 2019; 42: 101305.

45. Wang J, Wei W, Tang Q, et al. Oxysophocarpine suppresses hepatocellular carcinoma growth and sensitizes the therapeutic blockade of anti-Lag-3 via reducing FGL1 expression. Cancer Med. 2020; 9: 7125-36.

46. Gong C, Yu X, Zhang W, et al. Regulating the immunosuppressive tumor microenvironment to enhance breast cancer immunotherapy using pH-responsive hybrid membrane-coated nanoparticles. J Nanobiotechnology. 2021; 19: 58.

47. Sparks JA. Rheumatoid Arthritis. Ann Intern Med. 2019; 170: ITC1-ITC16.

48. Liu S, Guo Y, Lu L, et al. Fibrinogen-Like Protein 1 Is a Novel Biomarker for Predicting Disease Activity and Prognosis of Rheumatoid Arthritis. Front Immunol. 2020; 11: 579228.

49. Esfahani M, Baranchi M, Goodarzi MT. The implication of hepatokines in metabolic syndrome. Diabetes Metab Syndr. 2019; 13: 2477. 
50. Watt MJ, Miotto PM, De Nardo W, et al. The Liver as an Endocrine Organ-

Linking NAFLD and Insulin Resistance. Endocr Rev. 2019; 40: 1367-93.

51. Jensen-Cody SO, Potthoff MJ. Hepatokines and metabolism: Deciphering communication from the liver. Mol Metab. 2021; 44: 101138.

52. Xu M, Han Y, Liu G, et al. Preclinical Study of a Fully Human Anti-PD-L1 Antibody as a Theranostic Agent for Cancer Immunotherapy. Mol Pharm. 2018; 15: 4426-33. 